\title{
Editorial
}

\section{Nuclear Power Plants Safety and Maintenance}

\author{
Wael H. Ahmed, ${ }^{1}$ Atef Mohany, ${ }^{2}$ and Bing $\mathrm{Li}^{3}$ \\ ${ }^{1}$ School of Engineering, University of Guelph, Guelph, ON, Canada \\ ${ }^{2}$ Department of Automotive, Mechanical and Manufacturing Engineering, UOIT, ON, Canada \\ ${ }^{3}$ AMEC Nuclear Safety Solutions Ltd., Canada
}

Correspondence should be addressed to Wael H. Ahmed; ahmedw@uoguelph.ca

Received 17 June 2014; Accepted 17 June 2014; Published 6 July 2014

Copyright (C) 2014 Wael H. Ahmed et al. This is an open access article distributed under the Creative Commons Attribution License, which permits unrestricted use, distribution, and reproduction in any medium, provided the original work is properly cited.

With growing concerns about global warming, building more nuclear power plants is becoming one of the inevitable options to provide the world's electricity. This is mainly because nuclear power is considered as the major source of "carbon-free" energy. Currently, extensive preventive maintenance, inspection, and monitoring programs are developed to ensure safe and reliable operation of nuclear power plants. These programs involve safety analysis codes, equipment health monitoring, degradation-specific inspections, and plant lifecycle management (PLM). Although extensive effort has been invested to develop such programs, nuclear power plants continue to experience problems and therefore more accurate analysis and related research work still receive a great attention. This is mainly because the unresolved safety problems or lack in systems monitoring often causes significant safety-related events at nuclear power plants. Therefore, the demand for applied research work continues to grow in order to mitigate such events, increase the plant capacity factor, and gain public support for nuclear power as an alternative power source.

This special issue identifies and analyzes major practical problems in nuclear energy and it contains 7 articles out of 14 articles that were initially received for publication. Even with the limited number of articles presented in this special issue, a wide range of topics are covered to present different experiences from several nuclear power plants and research institutions around the world. These topics are gathered in this issue to present the best engineering analyses, research effort, and practice with as little prejudice as possible.

The issue starts by the novel method for optimization of the maintenance activities presented by A. Volkanovski and L. Cizelj in the nuclear power plant in order to improve plant safety. The authors consider different constraints on the optimization of the maintenance activities in the nuclear power plants. Three severe accident sequences of nuclear plants are discussed by G. Shao et al. to show that the natural circulation phenomenon results in heat transfer from the core to the pipes in RCS and consequently may cause the creep rupture of pipes in RCS and delay the severe accident progression. Also, S. Liu et al. were able to show that thermal stress failure caused by alternating operational loads is one of the important damage mechanisms in the nuclear power plants. Furthermore, R. K. Ur et al. discuss the impact of instrumentation and control components on the architecture of reactor protection systems.

On the numerical and statistical analysis side, the article of S. U.-D. Khan et al. presents a modification to the neutron kinetics code, which originally works for the cylindrical type fuel elements, to plate type fuel nuclear reactor. Moreover, the study performed by M. El Nakla et al. has stressed the importance of the local scale observation leading to better understanding of the $\mathrm{CHF}$ phenomena in large pipe. They were able to predict the occurrence of critical heat flux in horizontal tubes using an extra correction factor to account for flow stratification. Finally, T. H. Woo contributed to this special issue with a paper on the nuclear power plant security. He presented dynamical assessment, which helps operator to find the dangerous situation in the site and avoid possible incidents that could be a catastrophe for the NPPs. We believe that this special issue provides interesting information on the recent progress in nuclear power research.

$$
\begin{array}{r}
\text { Wael H. Ahmed } \\
\text { Atef Mohany } \\
\text { Bing Li }
\end{array}
$$




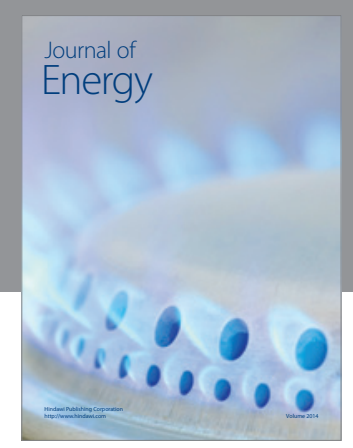

Journal of

Industrial Engineering
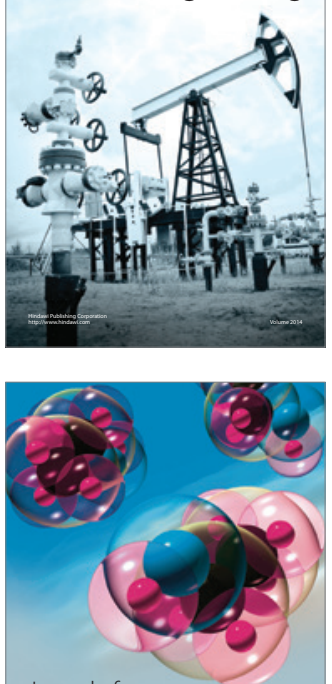

Fuels
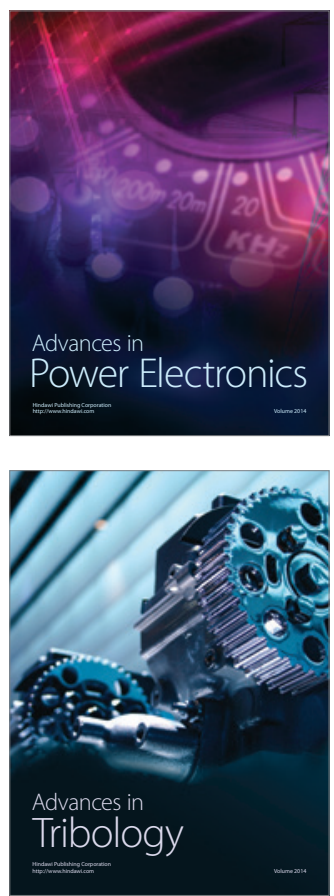

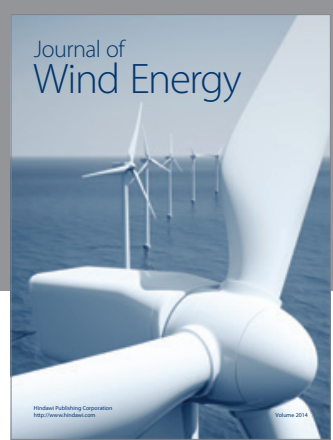

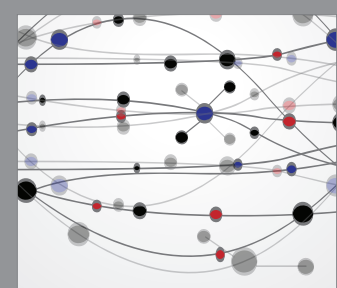

The Scientific World Journal

Submit your manuscripts at http://www.hindawi.com

Journal of

Structures
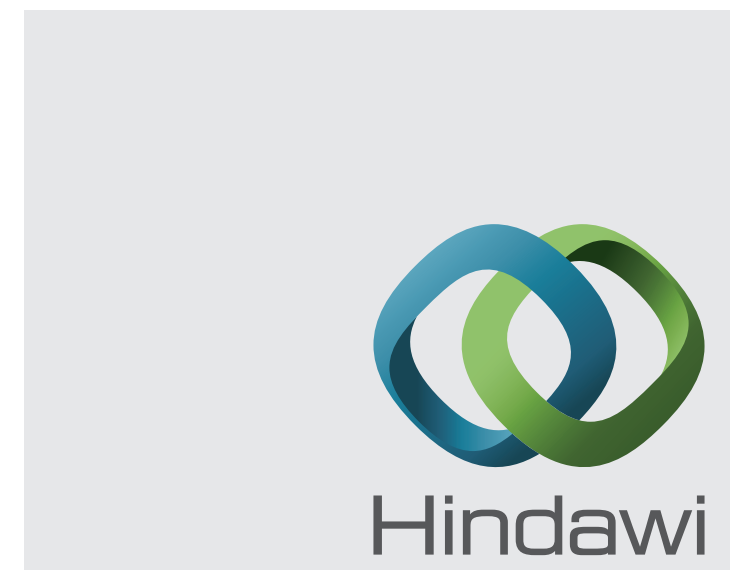

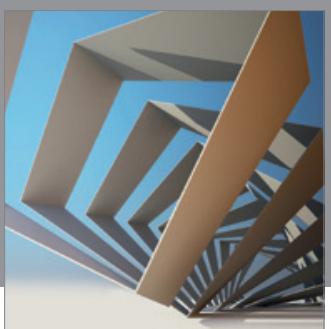

Rotating

Machinery
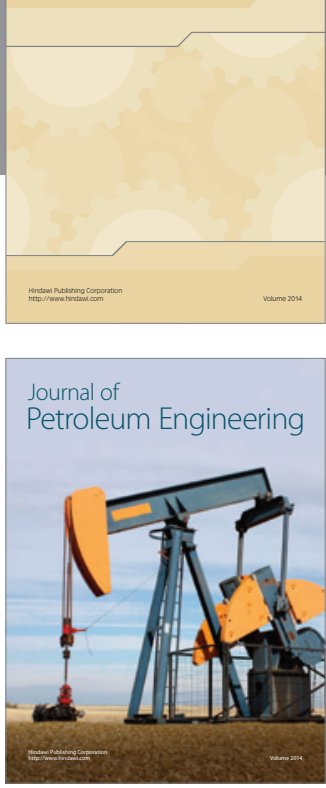

Journal of

Solar Energy
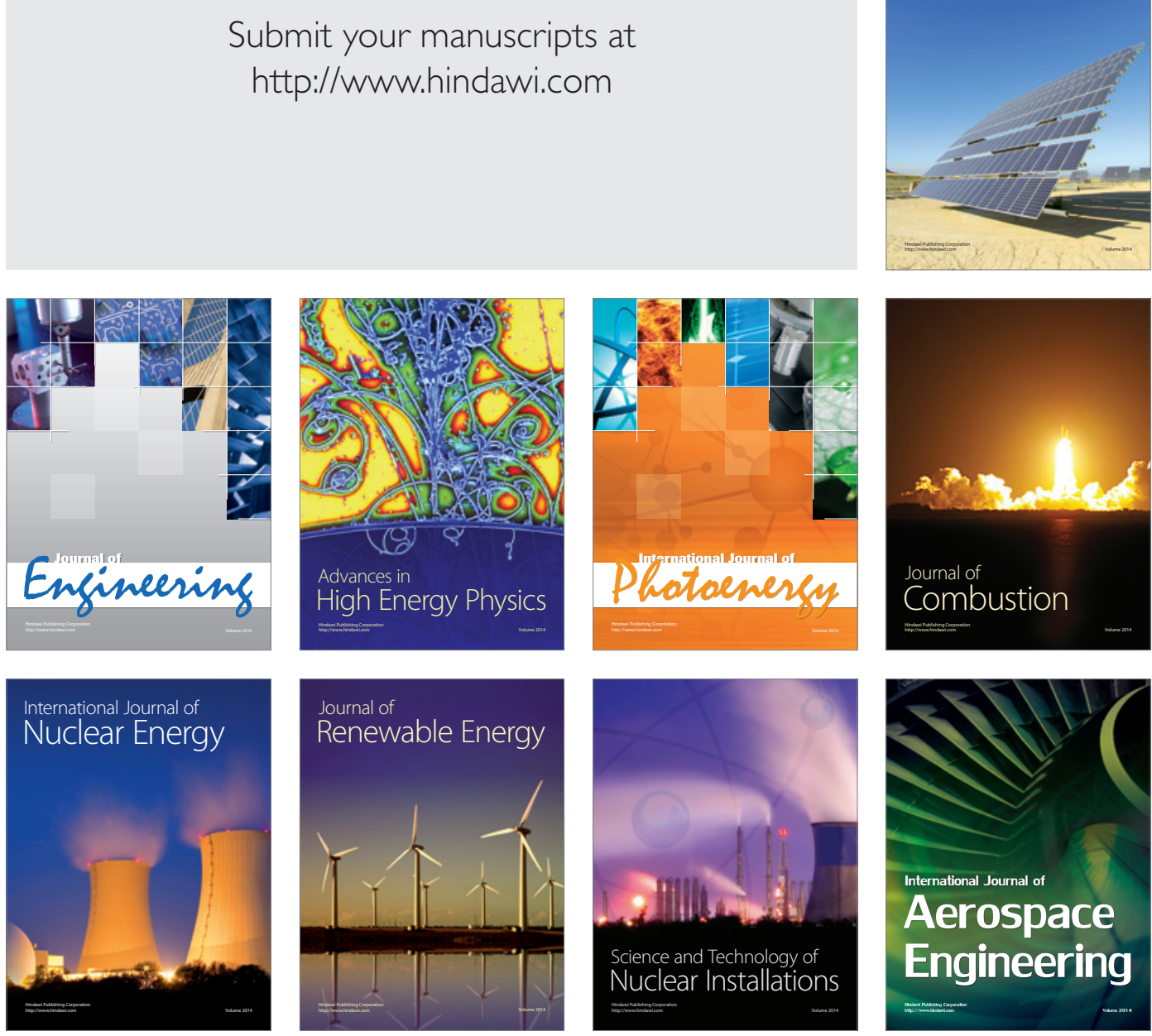